\title{
Id1 induces tubulogenesis by regulating endothelial cell adhesion and cytoskeletal organization through $\beta 1$-integrin and Rho-kinase signalling
}

\author{
JUHUI QIU ${ }^{1}$, GUIXUE WANG ${ }^{1}$, QIN PENG ${ }^{1}$, JIANJUN HU $^{1}$, XIANGDONG LUO ${ }^{2}$, \\ YIMING ZHENG ${ }^{1}$, YANQUN TENG ${ }^{1}$ and CHAOJUN TANG $^{1}$ \\ ${ }^{1}$ Key Laboratory of Biorheological Science and Technology, Ministry of Education, Chongqing Engineering Laboratory \\ in Vascular Implants, Bioengineering College of Chongqing University; ${ }^{2}$ Burn Research Institute, \\ Southwest Hospital, Third Military Medical University, Chongqing 400044, P.R. China
}

Received March 30, 2011; Accepted May 16, 2011

DOI: $10.3892 /$ ijmm.2011.741

\begin{abstract}
The inhibitor of differentiation 1 (Id1) protein is required for tubulogenesis, but the molecular signalling pathways remain unclear. Overexpression (Id1-t) or downregulation (si-Id1) of Id-1 in cell lines, were used to study the function of Id1. The expression of Id1 and $\beta 1$-integrin was assessed by Western blotting. Up-regulation of Id1 in human umbilical vascular endothelial cells (HUVECs) activated the expression of $\beta 1$-integrin and promoted cell adhesion and spreading. Conversely, down-regulation of Id1 suppressed $\beta 1$-integrin expression and inhibited tubulogenesis. By using a $\beta 1$-integrin antibody to inhibit $\beta 1$-integrin function, we demonstrated that Id1-induced cell adhesion and tubulogenesis were mediated by $\beta 1$-integrin. In addition, HUVECs overexpressing Id1 were able to promote capillary tube formation through cytoskeleton reorganization and cell contraction. Finally, the Rho-kinase inhibitor Y27632 inhibited tubulogenesis induced by Id1. Our findings provide evidence that Id1 regulates tubulogenesis in vitro through $\beta 1$-integrin and Rho-kinase signalling.
\end{abstract}

\section{Introduction}

Controlled angiogenesis is vital for normal organ development and tissue repair, while angiogenesis can sustain tumorigenesis and age-related macular degeneration (1). In addition, angiogenesis is a key event in the pathogenesis of some forms of cardiovascular disease (2). Although angiogenesis has been

Correspondence to: Dr Guixue Wang, Key Laboratory of Biorheological Science and Technology, Ministry of Education, Chongqing Engineering Laboratory in Vascular Implants, Bioengineering College of Chongqing University, Chongqing 400044, P.R. China

E-mail: wanggx@cqu.edu.cn

Key words: inhibitor of differentiation 1, tubulogenesis, cytoskeletal organization, $\beta 1$-integrin, Rho-kinase identified as a key development in many progressive diseases, the specific molecular mechanisms of angiogenesis are not well understood.

The four Id (inhibitor of differentiation or DNA binding) proteins (Id1-4) all contain a helix-loop-helix motif that inhibits the activity of basic helix-loop-helix transcription factors by restraining DNA binding. The Id1 protein has been implicated in the regulation of the cell cycle and in differentiation (3). Since the initial discovery that Id1 is required for angiogenesis (4), Id1 protein induced-angiogenesis has been studied in several experimental systems. Transplantation of mature endothelial cells overexpressing Id1 may serve as a novel and useful strategy for therapeutic angiogenesis (5). Studies have also indicated that the overexpression of Id1 protein was correlated with angiogenesis in tumors (6). Although the molecular mechanisms of Id1-mediated cell proliferation and apoptosis have been identified (7), little is known about the mechanism of Id1-mediated angiogenesis.

Angiogenesis is a complex event and requires a wellorchestrated integration of migration, proliferation, extracellular matrix (ECM) degradation, cell contraction, and survival. Previous studies reported that Id1 can regulate cell migration (5), proliferation (5) and ECM degradation (8). However, cellmatrix adhesion and F-actin cytoskeleton reorganization, which play crucial roles in endothelial cell adhesion during angiogenesis $(9,10)$, were absent. Moreover, cytoskeletal regulatory molecules are probably involved in angiogenesis (11). Furthermore, angiogenesis requires a polarized morphology with a single, broad lamellipod at the front that contains an actin cytoskeletal meshwork associated with many static cellmatrix adhesions (12). Therefore, the mechanisms underlying endothelial cell (EC) morphogenesis are likely to be critical for angiogenesis. We propose that Id1 regulates angiogenesis by remodelling cell-matrix adhesion and through cytoskeleton reorganization.

In the present study, we investigated whether Id 1 can regulate cell-matrix adhesion and the cell contractility of endothelial cells to control angiogenesis. We uncovered a novel signalling system whereby Id1 regulates angiogenesis through expression of $\beta 1$-integrin and activation of the Rho-kinase 
cascade; blockade of either $\beta 1$-integrin or of Rho-kinase suppressed Id1-induced angiogenesis.

\section{Materials and methods}

Materials and cell culture. Human umbilical vascular endothelial cells (HUVECs) were obtained from the American Type Culture Collection (ATCC, Manassas, VA), and were maintained in RPMI-1640 supplemented with $10 \%$ fetal bovine serum (FBS) at $37^{\circ} \mathrm{C}$ in $5 \% \mathrm{CO}_{2}$. The Rho-kinase blocker Y27632 was purchased from Sigma. Antibodies against Id1 and $\beta 1$-integrin (for $\beta 1$-integrin blocking antibody and $\beta 1$-integrin-FITC for flow cytometry) were purchased from Santa Cruz Biotechnology. The $\beta 1$-integrin protein used in Western blotting was purchased from Abcam. The VEGF antibody was purchased from Wuhan Boster Biological Technology (China).

Generation of Idl-t and si-Idl. The HUVECs-pIRES (con-t) and the HUVECs-Id1 transfectants (Id1-t) were established as previously described (13). Stable transfectants were selected with by cultivation with $800 \mu \mathrm{g} / \mathrm{ml}$ geneticin (G418, SigmaAldrich) after transfection. The surviving colonies were collected after 3 weeks.

A stable Id1 si-RNA vector was generated using a previously described method (14). Briefly, the primers with short hairpin RNA sequences targeting the Id1 coding region were cloned into the pSuppressor-Retro vector to generate the siRNA expression vector. The sequences of the si-Id1 primers were: si-Id1-F, TCG AGG CTG TTA CTC ACG CCT CAA GGA AGC TTG CTT GAG GCG TGA GTA ACA GCC TTT TT; si-Id1-R, CTA GAA AAA GGC TGT TAC TCA CGC CTC AAG CAA GCT TCC TTG AGG CGT GAG TAA CAG CC. The control vector was generated using the same procedures as the siRNA vector, and the short hairpin RNA sequence was replaced with nonsense sequences that are not homologous to the human genome. The sequences of the control primers (14) were: si-con-F, TCG AGC GTA TTG CCT AGC ATT ACG TGA TGC TTG ACG TAA TGC TAG GCA ATA CGC TTT TT; si-con-R, CTA GAA AAA GCG TAT TGC CTA GCA TTS CGT CAA GCT TCA CGT AAT GCT AGG CAA TAC GC. The resulting vectors were then transfected into HUVECs using Lipofectin (Life Technologies) in accordance with the manufacturer's instructions. Positive si-Id1 and si-con clones were then selected in neomycin $(200 \mu \mathrm{g} / \mathrm{ml})$ and stable transfectants were isolated after 3 weeks of drug selection.

Cell attachment and spreading assays. For cell attachment assays, cells were gently trypsinized and suspended in serumfree medium at $1.5 \times 10^{5}$ cells $/ \mathrm{ml}$ and $300 \mu \mathrm{l}$ of cell suspension was added to each well in 24-well plates. After $30 \mathrm{~min}$, unattached cells were removed by washing with PBS, and attached cells were treated with trypsin and counted with a haemocytometer.

To assess cell spread on collagen I gels, diluted collagen I $(500 \mu \mathrm{g} / \mathrm{ml})$ in serum-free medium was added to 12 -well plates $(500 \mu \mathrm{l} /$ well). After the diluted collagen had polymerized at $37^{\circ} \mathrm{C}, 1.2 \times 10^{5}$ cells were added to each well. Photographs were taken after $24 \mathrm{~h}$ and cell areas (spread) were quantified using the ImageTool software.
For antibody blocking analysis, the cells were mixed with $\beta 1$-integrin antibody $(10 \mu \mathrm{g} / \mathrm{ml})$ for $15 \mathrm{~min}$ and then plated at $1.2 \times 10^{5}$ cells/well.

Collagen gel contraction. For collagen gel contraction assays (11), $600 \mu \mathrm{l}$ cushions of $2 \mathrm{mg} / \mathrm{ml}$ collagen I were prepared in 24 -well plates. Next, HUVECs $\left(5 \times 10^{5} /\right.$ well $)$ were seeded in serum-free medium. After all cells had attached, the medium was removed and cells were overlaid with fresh medium containing $600 \mu 1$ of $2 \mathrm{mg} / \mathrm{ml}$ collagen I, which subsequently polymerized. Gel diameters were monitored with a dissecting microscope two days later.

Western immunoblotting. Cells were washed three times with ice-cold PBS, and lysates were prepared in buffer containing $1 \%$ NP-40, 0.5\% deoxycholate, $0.1 \%$ SDS, $10 \mathrm{mM}$ Tris-HCl, $0.2 \mathrm{mM}$ PMSF and protease inhibitors. Protein concentration was determined by the BCA method. Equal amounts of protein were loaded onto each lane of a $12 \%$ SDS-PAGE gel. After blotting, polyvinylidene difluoride membranes were blocked for $2 \mathrm{~h}$ in $5 \%$ milk powder in TBS-Tween at room temperature. Membranes were then incubated with primary antibodies overnight at $4^{\circ} \mathrm{C}$. The binding of secondary HRP antibodies was visualized by ECL or ECL Plus. The band of the Western blot analyses was quantified with Quantity One software. Normalizing for total cell protein was performed using $\beta$-actin.

Flow cytometry. The cell suspension was centrifuged at $1000 \mathrm{rpm}$ for $5 \mathrm{~min}$, and the cells resuspended in blocking buffer (49 ml RIPA-1640, $1 \mathrm{~g}$ BSA, $1 \mathrm{ml}$ fetal calf serum) on ice for $15 \mathrm{~min}$. Some cell pellets were treated with $\beta 1$-integrinFITC antibody ( $2 \mu \mathrm{g} / \mathrm{tube})$ except the control. The pellets were dispersed, mixed well, and incubated on ice for $1 \mathrm{~h}$ with agitation every $20 \mathrm{~min}$ to prevent cell sedimentation and clumping. The cells were washed by adding $3 \mathrm{ml}$ of wash buffer (100 ml RIPA-1640, $1 \mathrm{~g} \mathrm{BSA})$ to the tubes, and centrifuged at $1000 \mathrm{rpm}$ for $5 \mathrm{~min}$ at $4^{\circ} \mathrm{C}$. The supernatant was aspirated, and the cell pellet dispersed by flicking the tube. These steps were repeated. After a final wash, the cells were resuspended in the sample tube to a final concentration of $3 \times 10^{6}$ cells $/ \mathrm{ml}$ in wash buffer.

F-actin staining. Human umbilical vascular endothelial cells were washed three times with PBS, plated on glass coverslips, and fixed for $30 \mathrm{~min}$ in PBS with 3.7\% formaldehyde. Fixed cells were then treated with $0.1 \%$ Triton X-100 for $10 \mathrm{~min}$. The F-actin was directly stained with rhodamine-phalloidin (1:100) for $30 \mathrm{~min}$ after blocking in $1 \% \mathrm{BSA}$ for $30 \mathrm{~min}$ at room temperature. Cell nuclei were counterstained with 4', 6-diamidino-2-phenylindole (DAPI) in the mounting medium. Cells were washed three times with PBS and photographed using a confocal microscope.

In vitro tube formation. The in vitro tube formation (15) assay was performed using a matrigel basement membrane matrix (BD Biosciences). Matrigel, kept on ice, was placed in 24-well culture plates at $200 \mu \mathrm{l} /$ well. The plates were then incubated at $37^{\circ} \mathrm{C}$ for $30 \mathrm{~min}$ to allow the matrigel to solidify. HUVECs were seeded at $10^{4} \mathrm{cells} / \mathrm{well}$ on the top of the solidified matrigel, and the plate was incubated at $37^{\circ} \mathrm{C}$ for $24 \mathrm{~h}$. Tube formation 

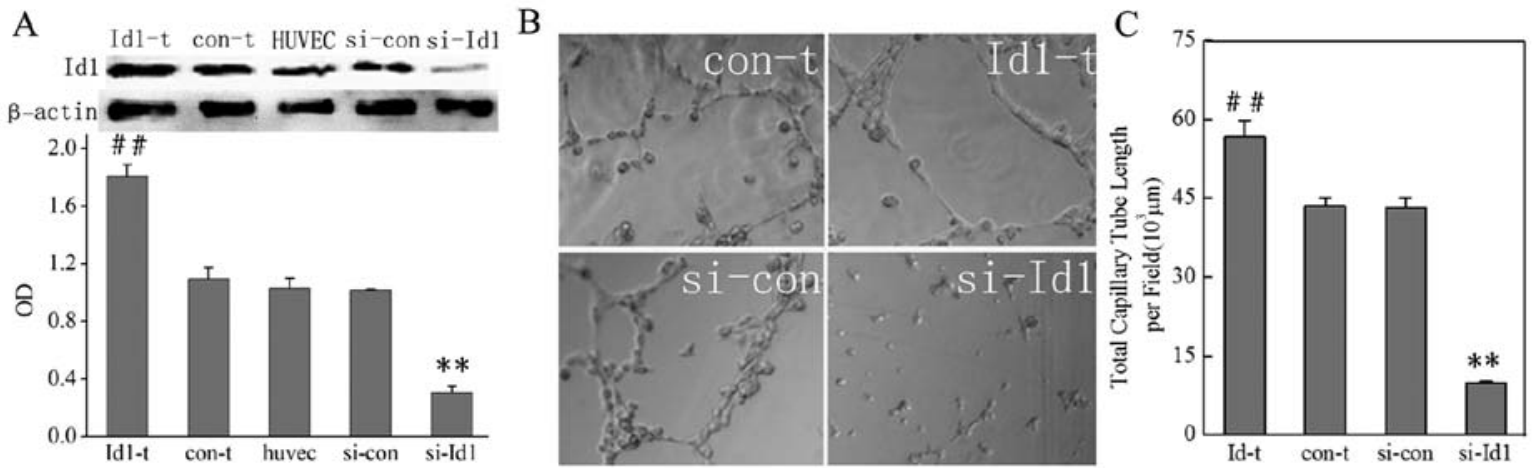

Figure 1. Overexpression of Id1 promotes angiogenesis in HUVECs in vitro. (A) The expression of Id1 protein in HUVECs with Id1-t, con-t, si-con and si-Id1 was examined by Western blotting. The data are relative to $\beta$-actin in the same lane. Each lane was loaded with $30 \mu \mathrm{g}$ of protein. (B) Tube formation ability of HUVECs cultured on matrigel gels with HUVECs expressing high and low levels of Id1. (C) Quantification of the total capillary tube length per field shown in $\mathrm{B} .{ }^{\# \#} \mathrm{P}<0.01$ for Id1-t vs. con-t; ${ }^{* *} \mathrm{P}<0.01$ for si-Id1 vs. si-con.

on matrigel was observed and analyzed under the microscope. The degree of angiogenesis was measured by multiplying the number of branch points by the total number of branches.

Statistical analysis. Data are given either as mean \pm SD or as proportions. In cases of significant differences between the groups, multiple comparisons between groups were made by a modified Student's t-test with the Bonferroni correction. Comparisons between 2 groups were made by independent t-test.

\section{Results}

Idl is required for endothelial cell tubulogenesis. To investigate the mechanisms of Id-mediated tubulogenesis, we established two transfectant clones, Id1 overexpression (Id1-t) and knockdown (si-Id1), and then investigated whether ectopic Id1 expression in HUVECs could lead to tubulogenesis. Results showed that Id1 expression in Id1-t clones was $~ 180 \%$ higher than that in control transfectant clones (con-t), while si-Id1 clones has just $30 \%$ Id1 expression compared to con-t clones (Fig. 1A). We then studied the tube forming ability of endothelial cells with higher or lower Id1 expression. The formation of capillary-like structures on matrigel by HUVECs was low in si-Id1 clones (Fig. 1B). Compared with control cells, there was a $30 \%$ increase in the length of tubulogenesis in Id1-t clones, while there was an $\sim 3$-fold decrease in si-Id 1 clones compared to control siRNA clones (Fig. 1C).

Idl is critical for cell adhesion and spreading. Cell adhesion and spread play critical roles in the angiogenic process. To determine whether Id1 protein was required for HUVECs adhesion, we cultured Id1-t, si-Id1, con-t and si-con clones on collagen-coated plates in serum-free medium. We found that Id1-t HUVECs adhered to the matrix more efficiently than control HUVECs, while the number of si-Id1 HUVECs adhering to collagen I was significantly decreased in the same time (Fig. 2A). Quantitative analysis of the number of adherent cells showed that Id1-t cells had a 2-fold higher number of adherent cells than the control group, while si-Id1 HUVECs had a 2-fold reduction in adherent cells (Fig. 2B). Cell area is critical for cell survival and cell function, so we examined the different cell spreading areas induced by Id1. Results showed that Id1-t HUVECs had a larger spreading area than control HUVECs, while si-Id1 HUVECs had a smaller spreading area on collagen I (Fig. 2C). Quantitative analysis of the cell area by ImageTool demonstrated that Id1-t HUVECs had a much larger spreading area than con-t clones, while si-Id1 HUVECs had a much smaller cell spreading area than either Id1-t or control clones (Fig. 2D).

Id1-induced cell adhesion and spreading is $\beta 1$-integrin dependent. In order to determine whether Id1 could induce endothelial cell adhesion by integrin-dependent ECM interactions, we examined the effect of Id1 on the expression of $\beta 1$-integrin in HUVECs. The Id1 overexpression led to the increased expression of $\beta 1$-integrin, while Id1 knockdown nearly abolished $\beta 1$-integrin expression (Fig. 3A). In addition, flow cytometry analysis indicated that Id1-t cells exhibited a 1.5 -fold induction of $\beta 1$-integrin protein compared with control ECs; however, si-Id1 cells showed a $60 \%$ reduction in $\beta 1$-integrin relative to control ECs (Fig. 3B).

To determine whether Id1-induced cell adhesion was dependent on the function of $\beta 1$-integrin, we treated cells with $\beta 1$-integrin antibody. This treatment resulted in a $75 \%$ inhibition of cell attachment. Although Id1 overexpression increased the EC attachment, the $\beta 1$-integrin antibody inhibited attachment of Id1-t cells to collagen I (Fig. 3C). These experiments indicate that the expression of $\beta 1$-integrin can be mediated by Id 1 and that Id1 also controlled cell adhesion to collagen I through the cell surface protein $\beta 1$-integrin.

In additional experiments, we used a $\beta 1$-integrin inhibitory antibody to examine the Id1-induced tubulogenesis in vitro. The results showed that capillary like-structures were abrogated by $\beta 1$-integrin antibody even in Id1-t cells (Fig. 3D), suggesting that Id1-induced cell adhesion, spread and tubulogenesis are $\beta 1$-integrin-dependent.

Id1 regulates EC actin stress fibre formation and contraction of collagen I gels. Previous reports have shown that $\beta 1$-integrin is vital for the disassembly of the actin cytoskeleton (16) and cytoskeleton is critical for cell contraction (17). Therefore, the next series of experiments were aimed at gaining further information about the role of Id1 in endothelial cell actin 

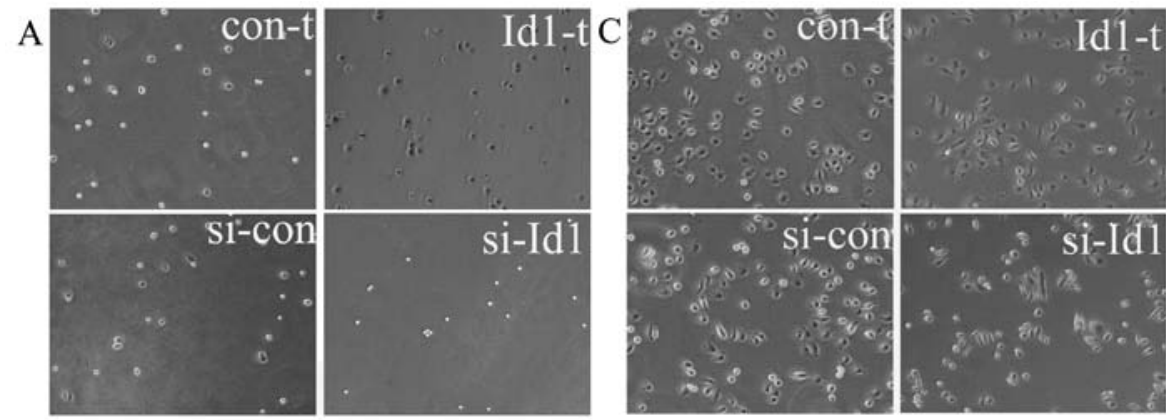

B

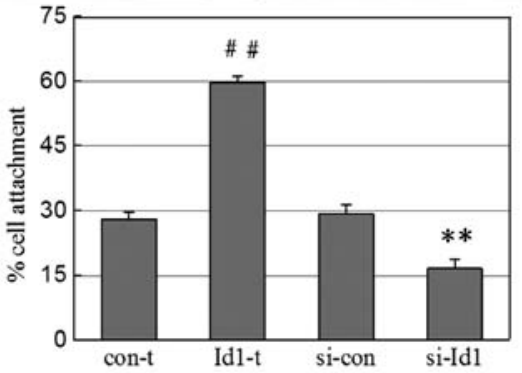

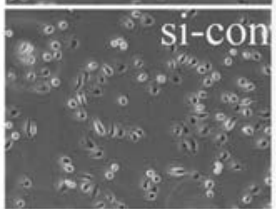

D

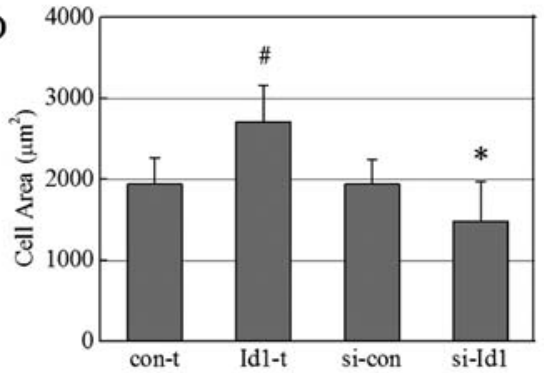

Figure 2. Overexpression of Id1 regulates HUVECs adhesion and spreading on type I collagen gels. (A) Adhesion of HUVECs with Id1-t, con-t, si-con and si-Id1 on type I collagen gels after $30 \mathrm{~min}$ (x100). (B) Quantification of the total number of the HUVECs adhered to the collagen I shown in (A). (C) Spreading of HUVECs with Id1-t, con-t, si-con and si-Id1 on type I collagen gels after $24 \mathrm{~h}$ (x100). (D) Quantification of the total number of the HUVECs adhered to the collagen I shown in $(\mathrm{C}) .{ }^{\# \#} \mathrm{P}<0.01$ and ${ }^{\#} \mathrm{P}<0.05$ for Id1-t vs. con-t; ${ }^{* *} \mathrm{P}<0.01$ and ${ }^{*} \mathrm{P}<0.05$ for si-Id1 vs. si-con.
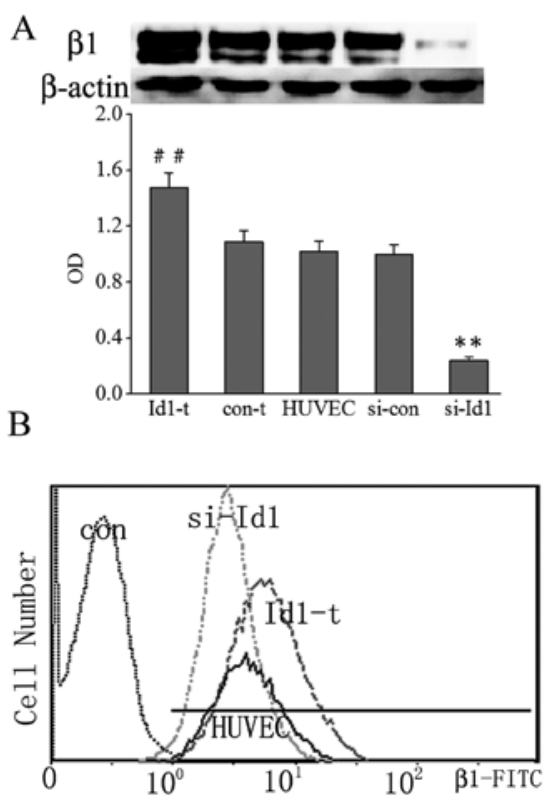
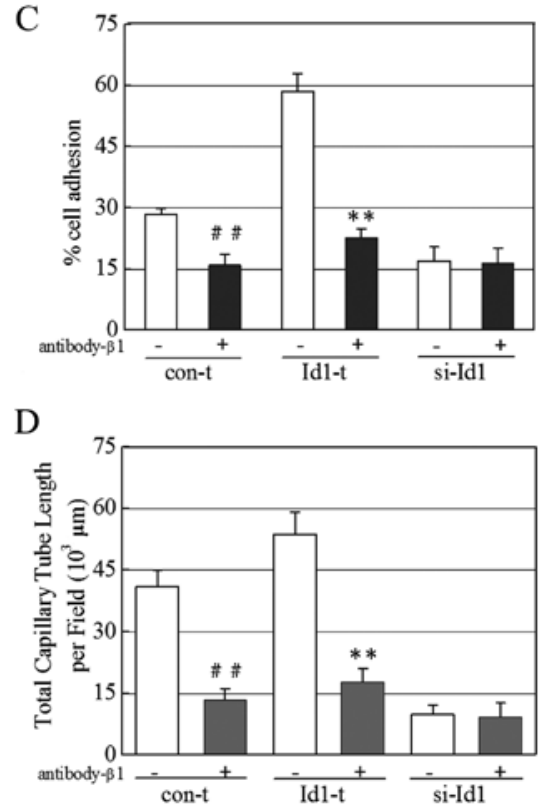

Figure 3. Overexpression of Id1 induces cell adhesion and tubulogenesis via regulating the expression of $\beta 1$-integrin. (A) Expression of $\beta 1$-integrin of HUVECs with Id1-t, con-t, si-con and si-Id1 were examined by Western blotting. ${ }^{\# \#} \mathrm{P}<0.01$ for Id1-t vs. con-t; ${ }^{* *} \mathrm{P}<0.01$ for si-Id1 vs. si-con. (B) Flow cytometry detected the effect of Id1-induced $\beta 1$-integrin expression. (C) Combination of $\beta 1$-integrin-blocking antibody (10 $\mu \mathrm{g} / \mathrm{ml})$ detected cell adhesion. (D) Combination of $\beta 1$-integrin antibody $(10 \mu \mathrm{g} / \mathrm{ml})$ detected cell tubulogenesis. ${ }^{\# \#} \mathrm{P}<0.01$ for con-t vs. con- $\mathrm{t}+\beta 1$-integrin antibody; ${ }^{* *} \mathrm{P}<0.01 \mathrm{for}$ Id1-t vs. Id1- $\mathrm{t}+\beta 1$-integrin antibody.

cytoskeleton organization and cell contraction. Cells overexpressing Id1 had more cytoplasmic F-actin and actin stress fibres than control cells, while si-Id1 cells had attenuated actin stress fibres and appeared tightly connected with a characteristic peripheral rim of F-actin (Fig. 4A). Actin stress fibres are critical for cell contraction; therefore, cell contractility induced by Id1 was assessed. Results showed that Id1 overexpression significantly increased the contraction of collagen I gels (Fig. 4B).
Idl-induced EC cytoskeleton and contraction depend on Rho-kinase. Rho GTPases are critical for regulating cytoskeleton organization, and have a prominent role in the control of cell migration and contraction (18). To investigate whether Rho-kinase is involved in Id1-enhanced endothelial contraction and tubulogenesis, we treated cells with $10 \mu \mathrm{M}$ of the highly specific Rho-kinase inhibitor, Y27632. This antagonist abolished the Id1-enhanced cytoskeleton formation completely and F-actin was strictly localized to the peripheral rim. The 
A
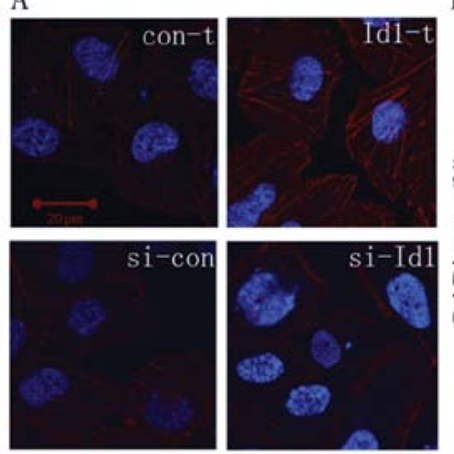

B

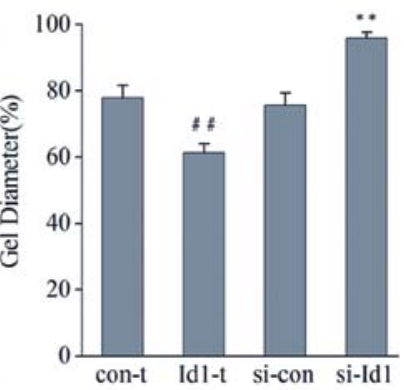

Figure 4. Id1 regulates HUVEC actin stress fiber formation and contraction of collagen I gels in vitro. (A) Rhodamine-phalloidin-staining of HUVECs with Id1-t, con-t, si-con and si-Id1. (B) The contraction of collagen I gels of HUVECs with Id1-t, con-t, si-con and si-Id1. ${ }^{\# \#} \mathrm{P}<0.01$ for Id1-t vs. con-t; ${ }^{* *} \mathrm{P}<0.01$ for si-Id1 vs. si-con.
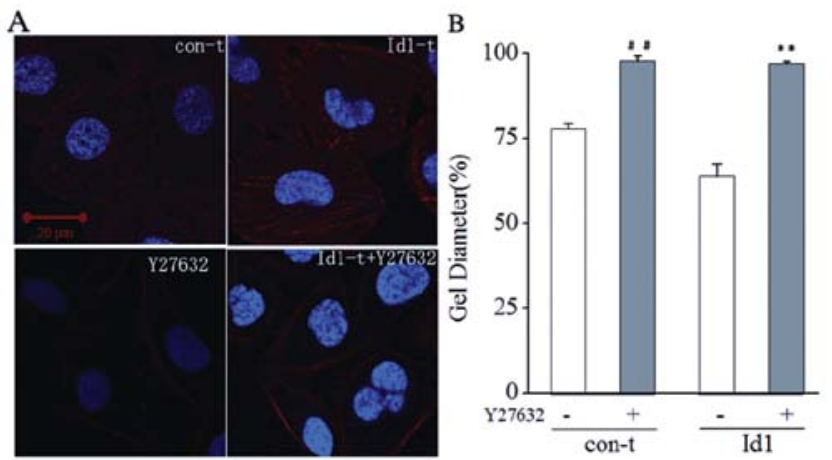

Figure 5. Id1-induced changes in the HUVEC cytoskeleton are dependent on RhoA and Rho-kinase signaling. (A) Rhodamine-phalloidin-staining of con-t and Id1-t, pre-treated for $1 \mathrm{~h}$ with $10 \mu \mathrm{mol} / 1$ Y27632 or untreated. (B) The contraction of collagen I gels of HUVECs with $10 \mu \mathrm{mol} / 1 \mathrm{Y} 27632$ or untreated. ${ }^{\# \#} \mathrm{P}<0.01$ for con- $\mathrm{t}+\mathrm{Y} 27632$ vs. con-t; ${ }^{* *} \mathrm{P}<0.01$ for $\mathrm{Id} 1-\mathrm{t}+\mathrm{Y} 27632$ vs. Id1-t.

cytoskeleton in control cells was also significantly altered (Fig. 5A). Results showed that Y27632 also prevented Id1-induced contraction of collagen I gels (Fig. 5B). These data indicate that inhibition of Rho-kinase not only prevented the formation of Id1-induced actin stress fibres but also reduced HUVECs contraction. We next used Y27632 to examine whether Id1-induced tubulogenesis was mediated through Rho-kinase activity. Indeed, the same dose of Y27632 also destroyed capillary network formation (Fig. 6). These data suggest that Id1-induced actin stress fibres and contractility were mediated by Rho-kinase signalling and by the tension generation by the cytoskeleton.

\section{Discussion}

The aim of this study was to investigate the molecular mechanisms of tubulogenesis mediated by Id1 protein. We characterized the mechanisms by which Id1 regulates tubulogenesis through the cell-matrix adhesion protein $\beta 1$-integrin and by cytoskeletal reorganization, leading to enhanced cell contractility. Both up-regulation of $\beta 1$-integrin and the cytoskeletal rearrangements/cell contractility associated with

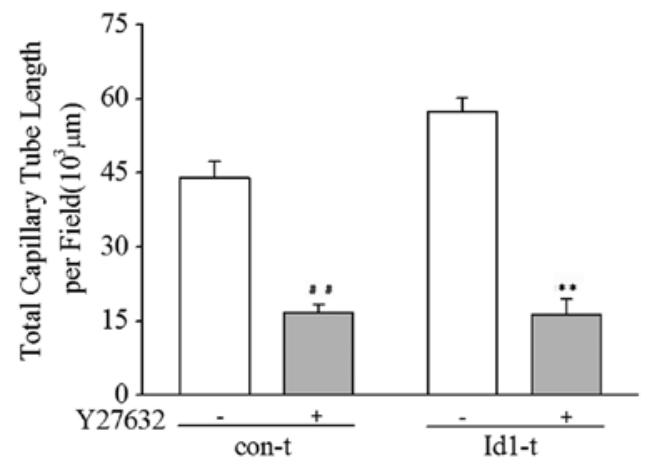

Figure 6. Effect of Y27632 on the Id1-induced tubulogenesis in HUVECs. Treatment of HUVECs with Y27632 $(10 \mu \mathrm{M})$, a RhoA inhibitor, inhibits of capillary tube formation ability induced by Id1 expression in HUVECs. ${ }^{\# \#} \mathrm{P}<0.01$ for con-t vs. con- $t+Y 27632 ;{ }^{* *} \mathrm{P}<0.01$ for Id1-t vs. Id1- $t+Y 27632$.

tubulogenesis were dependent on the Rho-kinase signalling cascade. This work demonstrates that Id1-induced tubulogenesis is associated with changes in cell adhesion and spreading, possibly mediated by $\beta 1$-integrin, and that Id 1 regulated the expression of $\beta 1$-integrin. The F-actin cytoskeleton and the Rho family kinases were involved in tubulogenesis induced by Id1 overexpression, and Id1 controlled cell contraction. Thus, our findings provide insight into the molecular mechanisms of Id1-regulated tubulogenesis.

Cell adhesion to ECM is a dynamic process involving attachment, spread, and formation of focal adhesions and stress fibres that generate a strong adhesive state (19). The strong adhesive state is characteristic of a differentiated, quiescent cell, whereas cells in the intermediate adhesive state include those involved in responding to injury during wound healing or tissue remodelling (20). Cell shape can regulate cell fate and function (21) and actin cytoskeleton is critical for cell volume (22). In the present study, we found that Id1 protein could induce cell adhesion and spreading. Therefore, Id1-induced cell adhesion and spreading could contribute to the Id1 related tubulogenesis.

Integrins, cytoskeletal proteins, and focal adhesion kinase (FAK) are critical for cell adhesion in three-dimensional (3D) matrices derived from tissues or in $2 \mathrm{D}$ cell culture. $\beta 1$-integrin is essential for EC adhesion, migration, and survival during tubulogenesis (23). Animal models have demonstrated that $\beta 1$-integrin is a critical adhesion molecule to induce therapeutic tubulogenesis in cell-based therapy (24). The Id1 and $\beta 1$-integrin proteins are well known for their role in regulating tubulogenesis, but up to now no research has shown that Id1 induces the expression of $\beta 1$-integrin and that Id1-induced tubulogenesis is dependent on $\beta 1$-integrin. The mechanism by which Id1 regulates the expression of $\beta 1$-integrin is still not clear. Recently, it was reported that p53 expression may regulate the recycling of $\beta 1$-integrin (25), and Id1 can regulate the expression of p53 (26). We propose that the interaction of Id1 and p53 is essential for Id1-mediated expression of $\beta 1$-integrin, a possibility that will be addressed in future study.

The Rho family of small GTPases is involved in a series of complex biochemical networks that regulate cytoskeletal dynamics, morphogenesis, polarity, movement and cell division (27). Reorganization of the F-actin cytoskeleton is critical for endothelial cell migration and tubulogenesis (9). 
Limiting the formation of actin stress fibres and focal contacts in the adhering endothelial cells, and inhibition of adhesion, suppresses their angiogenic properties $(16,19)$. The organization of the F-actin (as well as the microtubule) network and formation of cell-matrix adhesions in response to extracellular cues are controlled by small GTPases of the Rho family (18). Signalling by Rho/ROCK was shown to be an important mediator of a number of angiogenic processes induced by VEGF (11). This is consistent with our in vitro results that Id1 expression influenced the F-actin cytoskeleton during tubulogenesis and that Y27632 efficiently abolishes adhesion and migration of endothelial cells and capillary tube formation induced by Id1. The functional significance of F-actin cytoskeletal rearrangement and Rho family proteins in modulating Id1-mediated tubulogenesis has not been previously reported. Modulation of tubulogenesis by Id1 appears to depend on reorganization of the F-actin cytoskeleton mediated by Rho family signalling.

Our results have several important implications. The mechanisms through which Id1 regulates the expression of $\beta 1$-integrin are currently unknown. We showed that Id1-induced tubulogenesis by cell adhesion and cytoskeletal reorganization. This finding may prove critical for studies on how mechanical stimuli regulate cytoskeletal organization. Indeed, the expression of Id1 can be regulated by mechanical stimuli (28).

In conclusion, our current investigation identifies a novel mechanism for Id1-induced tubulogenesis: Id1 induces tubulogenesis by regulating endothelial cell adhesion and cytoskeletal organization through $\beta 1$-integrin and Rho-kinase signaling. Id1-induced tubulogenesis was dependent on $\beta 1$-integrin and corresponding changes in cell adhesion and spreading. This link should be further explored and may provide clues for the management of tubulogenesis related disease. In particular, Id1-induced tubulogenesis by cell adhesion and cytoskeletal reorganization demonstrated that Id1 can be used either to suppress or enhance cell adhesion by $\beta 1$-integrin and cell cytoskeletal reorganization by Rho-kinase. This study may lay a foundation for new rational therapeutic strategies to control new blood vessel formation.

\section{Acknowledgements}

This study was supported by grants from The National Natural Science Foundation of China (3097072, 30730093) and The Fundamental Research Funds for the Central Universities (CDJXS10 2322 10).

\section{References}

1. Kim WY and Lee HY: Brain angiogenesis in developmental and pathological processes: mechanism and therapeutic intervention in brain tumors. FEBS J 276: 4653-4664, 2009.

2. Drinane M, Mollmark J, Zagorchev L, et al: The antiangiogenic activity of rPAI-1(23) inhibits vasa vasorum and growth of atherosclerotic plaque. Circ Res 104: 337-345, 2009.

3. Sikder HA, Devlin MK, Dunlap S, et al: Id proteins in cell growth and tumorigenesis. Cancer Cell 3: 525-530, 2003.

4. Lyden D, Young AZ, Zagzag D, et al: Id1 and Id 3 are required for neurogenesis, angiogenesis and vascularization of tumour xenografts. Nature 401: 670-677, 1999.

5. Nishiyama K, Takaji K, Kataoka K, et al: Id1 gene transfer confers angiogenic property on fully differentiated endothelial cells and contributes to therapeutic angiogenesis. Circulation 112: 2840-2850, 2005.
6. Kim HJ, Chung H, Yoo YG, Kim H, Lee JY, Lee MO and Kong G: Inhibitor of DNA binding 1 activates vascular endothelial growth factor through enhancing the stability and activity of hypoxia-inducible factor-1alpha. Mol Cancer Res 5: 321-329, 2007.

7. Wong Y, Wang X and Ling MT: Id-1 expression and cell survival. Apoptosis 9: 279-289, 2004.

8. Sun X, Li C, Zhuang C, Gilmore WC, Cobos E, Tao Y and Dai Z: Abl interactor 1 regulates Src-Id1-matrix metalloproteinase 9 axis and is required for invadopodia formation, extracellular matrix degradation and tumor growth of human breast cancer cells. Carcinogenesis 30: 2109-2116, 2009.

9. van Nieuw Amerongen GP, Koolwijk P, Versteilen A and van Hinsbergh VW: Involvement of RhoA/Rho kinase signaling in VEGF-induced endothelial cell migration and angiogenesis in vitro. Arterioscler Thromb Vasc Biol 23: 211-217, 2003.

10. Liebl J, Weitensteiner SB, Vereb G, Takacs L, Furst R, Vollmar AM and Zahler S: Cyclin-dependent kinase 5 regulates endothelial cell migration and angiogenesis. J Biol Chem 285: 35932-35943, 2010.

11. Hoang MV, Whelan MC and Senger DR: Rho activity critically and selectively regulates endothelial cell organization during angiogenesis. Proc Natl Acad Sci USA 101: 1874-1879, 2004.

12. Danen EH, van Rheenen J, Franken W, Huveneers S, Sonneveld P, Jalink $\mathrm{K}$ and Sonnenberg A: Integrins control motile strategy through a Rho-cofilin pathway. J Cell Biol 169: 515-526, 2005.

13. Chen L, Qiu J, Yang C, Yang X, Chen X, Jiang J and Luo X: Identification of a novel estrogen receptor $\beta 1$ binding partner, inhibitor of differentiation-1, and role of ER $\beta 1$ in human breast cancer cells. Cancer Lett 278: 210-219, 2009.

14. Ling MT, Lau TC, Zhou C, et al: Overexpression of Id-1 in prostate cancer cells promotes angiogenesis through the activation of vascular endothelial growth factor (VEGF). Carcinogenesis 26: 1668-1676, 2005

15. Sakurai D, Tsuchiya N, Yamaguchi A, et al: Crucial role of inhibitor of DNA binding/differentiation in the vascular endothelial growth factor-induced activation and angiogenic processes of human endothelial cells. J Immunol 173: 5801-5809, 2004.

16. Bix G, Fu J, Gonzalez EM, et al: Endorepellin causes endothelial cell disassembly of actin cytoskeleton and focal adhesions through alpha2 $\beta 1$ integrin. J Cell Biol 166: 97-109, 2004.

17. Koltsova SV, Gusakova SV, Anfinogenova YJ, Baskakov MB and Orlov SN: Vascular smooth muscle contraction evoked by cell volume modulation: role of the cytoskeleton network. Cell Physiol Biochem 21: 29-36, 2008.

18. Etienne-Manneville $\mathrm{S}$ and Hall A: Rho GTPases in cell biology. Nature 420: 629-635, 2002

19. Cazes A, Galaup A, Chomel C, et al: Extracellular matrix-bound angiopoietin-like 4 inhibits endothelial cell adhesion, migration, and sprouting and alters actin cytoskeleton. Circ Res 99: 1207-1215, 2006.

20. Murphy-Ullrich JE: The de-adhesive activity of matricellular proteins: is intermediate cell adhesion an adaptive state? J Clin Invest 107: 785-790, 2001

21. Kilian KA, Bugarija B, Lahn BT and Mrksich M: Geometric cues for directing the differentiation of mesenchymal stem cells. Proc Natl Acad Sci USA 107: 4872-4877, 2010.

22. Papakonstanti EA, Vardaki EA and Stournaras C: Actin cytoskeleton: a signaling sensor in cell volume regulation. Cell Physiol Biochem 10: 257-264, 2000.

23. Carlson TR, Hu H, Braren R, Kim YH and Wang RA: Cellautonomous requirement for $\beta 1$ integrin in endothelial cell adhesion, migration and survival during angiogenesis in mice. Development 135: 2193-2202, 2008.

24. Li TS, Ito H, Hayashi M, Furutani A, Matsuzaki M and Hamano K: Cellular expression of integrin- $\beta 1$ is of critical importance for inducing therapeutic angiogenesis by cell implantation. Cardiovasc Res 65: 64-72, 2005.

25. Muller PA, Caswell PT, Doyle B, et al: Mutant p53 drives invasion by promoting integrin recycling. Cell 139: 1327-1341, 2009.

26. Kim H, Chung H, Kim HJ, Lee JY, Oh MY, Kim Y and Kong G: Id-1 regulates $\mathrm{Bcl}-2$ and $\mathrm{Bax}$ expression through $\mathrm{p} 53$ and NF-kappaB in MCF-7 breast cancer cells. Breast Cancer Res Treat 112: 287-296, 2008.

27. Jaffe $A B$ and Hall A: Rho GTPases: biochemistry and biology Annu Rev Cell Dev Biol 21: 247-269, 2005.

28. Ni CW, Qiu H, Rezvan A, et al: Discovery of novel mechanosensitive genes in vivo using mouse carotid artery endothelium exposed to disturbed flow. Blood 116: E66-E73, 2010. 